\title{
Link Scheduling Scheme with Shared Links and Virtual Tokens for Industrial Wireless Sensor Networks
}

\author{
Sergio Montero, Javier Gozalvez, Miguel Sepulcre \\ UWICORE, Ubiquitous Wireless Communications Research Laboratory \\ Universidad Miguel Hernández de Elche (UMH) \\ Elche (Alicante), Spain \\ smontero@umh.es,j.gozalvez@umh.es,msepulcre@umh.es
}

\begin{abstract}
Industrial wireless sensor networks can help improve the efficiency, reconfigurability and flexibility of future factories, and facilitate the introduction of new applications. Industrial applications are generally characterized with strict reliability and latency requirements. The capacity to meet such requirements is highly dependent on an efficient utilization of communication links. Such efficient utilization will become even more critical as the number of deployed sensors and traffic in factories increase. In this context, this paper presents a novel link scheduling scheme for industrial wireless sensor networks that uses shared links among nodes that are part of the same path or multi-hop route. The transmission of a message along a route acts as a virtual token to identify which node should use the shared links at each point in time. This study demonstrates that the proposed link scheduling scheme can significantly improve the reliability, latency and efficiency of industrial wireless sensor networks. The proposed link scheduling scheme is here applied to industrial wireless sensor networks, but it can be used in other centralized TDMA-based multi-hop wireless networks.
\end{abstract}

Keywords Industrial wireless sensor networks, scheduling, link scheduling, WirelessHART, ISA100.11a, shared links, virtual tokens, centralized TDMA, TDMA, multi-hop wireless networks, reliability, latency, energy consumption, factories of the future, industry 4.0, industrial wireless communications, industrial wireless networks.

\section{Introduction}

Industrial Wireless Sensor Networks (IWSNs) are expected to play a significant role in the design and operation of Factories of the Future (FoF) or the Industry 4.0. IWSNs can help reduce the cost and time needed for the installation and maintenance of cables and machinery, enhance the flexibility and reconfigurability of a factory, facilitate the introduction of healthcare solutions, and enable the connectivity of mobile subsystems or devices [1]. Significant advances have been achieved so far with the development of the WirelessHART [2] and ISA100.11a [3] standards for industrial automation and control applications. However, IWSNs still face significant challenges to ubiquitously guarantee the reliability and latency requirements of industrial applications [4].

WirelessHART and ISA100.11a are based on the IEEE 802.15.4 physical and MAC (Medium Access Layer) layers, and share some fundamental wireless technologies and mechanisms [5], e.g. a centralized network management and Time Division Multiple Access (TDMA) combined with Frequency Hopping (FH) [6]. Time slots are 10ms long in WirelessHART, and between 10 and $12 \mathrm{~ms}$ long in ISA100.11a. The duration of time slots is sufficient to allow for the transmission of a packet and the reception of an acknowledgment (the maximum packet size is 133 bytes, including headers). Time slots are organized into superframes. The number of time slots within a superframe indicates its periodicity. WirelessHART and ISA100.11a can define multiple superframes with different numbers of time slots to support different transmission intervals. A communications link (or simply a link) is defined by a timeslot offset within a superframe and a frequency channel. WirelessHART and ISA100.11a consider up to 15 different frequency channels (16 for ISA100.11a in some regions of the world). In both standards, a Network Manager (NM) is in charge of identifying and establishing the path or multi-hop route between source and destination nodes when a direct communication is not possible. The NM is also in charge of assigning the links for each hop of the path, which includes 
determining which nodes in each path should be assigned additional links to handle potential retransmissions. The NM has to assign the links ensuring that two nodes will not transmit simultaneously on the same link (i.e. on the same time slot and frequency channel). The scheduling scheme is hence critical to ensure the reliable and low latency delivery of messages in IWSNs.

The link scheduling process is particularly critical and challenging in industrial environments as many sensors periodically generate traffic with strict reliability and latency requirements. It is important noting that the WirelessHART and ISA100.11a standards do not specify any link scheduling scheme, but define instead a number of requirements that must be fulfilled [2]. One of such requirements for WirelessHART is that the scheduling scheme must assign at least three links per hop for attempting to transmit each message before its given transmission deadline. The first two attempts (i.e., the original transmission and one retransmission) utilize the same main path or route between the source and destination nodes. The third transmission attempt should be done over an alternative path. When scheduling links for a path, early hops should be scheduled first. The WirelessHART standard also specifies that no device can be scheduled to be in reception mode in two different channels during one time slot. Standards such as WirelessHART and ISA100.11a assign each node a fixed number of links per hop to transmit a message. The utilization of these links depends on the link quality conditions and on the number of retransmissions needed per transmitted message. A fixed assignment of links per hop might not be able to ensure high reliability levels if some hops experience particularly bad link quality conditions. It can also result in unnecessarily high latency and in an inefficient utilization of the links under good radio link quality conditions.

In this context, this paper proposes a novel link scheduling scheme that efficiently assigns and utilizes links in multi-hop IWSN communications. The proposed scheme is based on the concepts of shared links and virtual tokens. The proposed scheme uses shared links among nodes that are part of the same path or multi-hop route. The transmission of a message in the path acts as a virtual token to identify which node is allowed to transmit at each point in time in the shared links. This approach allows for a flexible assignment and use of the links, and therefore a better capacity to combat transmission errors and reduce latency. The conducted study demonstrates that the proposed scheme can improve the reliability and latency performance compared to existing link scheduling schemes for IWSNs. In addition, the proposed scheme makes a more efficient use of resources (links and energy). The proposed link scheduling scheme is here applied to IWSN, but it is important emphasizing that it can be used in other centralized TDMA-based multi-hop wireless networks.

The rest of the paper is organized as follows. Section 2 reviews the state of the art on scheduling in IWSNs. Section 3 describes the proposed link scheduling scheme, and Section 4 describes the metrics utilized to evaluate its performance. Section 5 presents analytical performance models for the different link scheduling schemes. In particular, it presents analytical expressions of the reliability and latency performance. Section 6 presents the results obtained with the proposed scheme, and compares its performance with different existing link scheduling schemes. The main contributions and conclusions of this study are summarized in Section 7.

\section{State of The Art}

Centralized and decentralized scheduling schemes have been proposed in the literature for multi-hop Wireless Sensor Networks (WSNs) [7][8]. Decentralized schemes rely on nodes with certain intelligence and partial knowledge of their neighborhood and network status. Such knowledge is utilized for distributed decision making processes. Decentralized schemes can in principle better react to changes in the network operation and dynamics [9]. Many decentralized proposals have been designed to minimize the average time needed to transmit a message between source and destination. For example, the scheme presented in [10] exploits information obtained by nodes from their local neighborhood to assign links without any transmission collisions. A different approach is followed by the decentralized scheme presented in [11] where the destination node is responsible for assigning links based on the characteristics of the traffic generated by the source node. The decentralized schemes generally offer more flexibility and capacity to adapt to network conditions than their centralized counterparts. However, centralized schemes are more suitable for IWSNs that have strict reliability and latency requirements [4] and utilize a centralized architecture and network management [12].

In centralized schemes, all scheduling decisions are taken by a central node (e.g. the Network Manager in WirelessHART), and the remaining nodes are only responsible for the radio transmission and reception of messages, and some limited data processing functions. It is important to remember that standards such as WirelessHART do not include or define any scheduling scheme, but define instead a number of requirements that must be fulfilled. Different centralized scheduling schemes have been reported in the literature. For example, the study in [13] proposes a graph route-based link scheduling scheme to handle transmission errors using retransmissions and route diversity. A graph is a collection of paths among network nodes. The graph identifies more than one route between any pair of source and destination nodes. A different approach is reported in [14] for the case in which it is necessary to assign links for the transmission of messages between a set of source and destination nodes (not a single pair). The scheduling schemes proposed in [14] seek ensuring that all transmission deadlines are met while avoiding conflicts between the 
concurrent transmissions. Most of the proposed centralized scheduling schemes address the link scheduling problem considering multiple possible paths between source and destination. When focusing on a single path, most of these schemes follow the concepts defined by the link scheduling schemes reported in [15] or [16]; these two schemes will hence be considered as reference schemes in this study.

The centralized link scheduling scheme presented in [15] assigns consecutively all the links to be utilized at each hop of a path. This includes the initial link to transmit the message from one node to the next one in the path, and the additional link for a potential retransmission of the message should the first transmission fail. This scheme is hence referred to as HbH (Hop by Hop). To illustrate its operation, we consider the example depicted in Fig. 1a. The example represents a main path of three hops between the source and destination nodes (the number of hops is represented by the variable $H$ ). WirelessHART indicates that two links should be assigned to each hop in the main path between source and destination. Following this requirement, Fig. $1 \mathrm{~b}$ represents the scheduling of links resulting from the application of $\mathrm{HbH}$. The first link is assigned for the initial transmission of the message, and the second one for its potential retransmission should the initial transmission fail. In this case, the transmission of a message from node $\mathrm{A}$ to node $\mathrm{B}$ will utilize the first link $l_{1}$. The following link $l_{2}$ is reserved for the possible retransmission of the same message from node A to node B in case the initial transmission was not successfully received at node B. Following the same principle, the following two links are reserved for the hop between node B and node $\mathrm{C}$ and so on. The scheme presented in [15] successfully delivers the message to the destination node as long as the number of transmission errors per hop is smaller than the number of links reserved to each hop. However, the sequential programming of all the links assigned to each hop increases the latency even in the absence of transmission errors.

An alternative scheme is presented in [16]; the scheme is here referred to as RTE. The scheme first assigns the links for the initial transmission of a message at each hop, and then the links for the possible retransmissions. Considering the example depicted in Fig. 1a, RTE assigns the first link $l_{1}$ to the transmission of a message from node A to node B (Fig. 1c). Link $l_{2}$ is assigned for the transmission of the same message from node $\mathrm{B}$ to node $\mathrm{C}$ and so on. Links $l_{4}, l_{5}$ and $l_{6}$ are then reserved for possible retransmissions of the message from node $\mathrm{A}$ to node $\mathrm{B}$, from node $\mathrm{B}$ to node $\mathrm{C}$, and from node $\mathrm{C}$ to node $\mathrm{D}$ respectively. This scheme reduces the time to deliver a message in the absence of transmission errors, but also the reliability in the delivery of the message to the destination node. For example, if the transmission of the message from node A to node B using $l_{1}$ fails, links $l_{2}$ and link $l_{3}$ cannot be utilized since the retransmission of the message from node A to node B (link $l_{4}$ ) is programmed after $l_{2}$ and $l_{3}$. If the retransmission using $l_{4}$ is successful, nodes $\mathrm{B}$ and $\mathrm{C}$ will only have one attempt each (using links $l_{5}$ and $l_{6}$ ) to successfully transmit the message to node D.

The schemes reported in [15] and [16] offer advantages in terms of reliability or latency. However, as pointed out in [6], the capacity to jointly guarantee end-to-end reliability and latency for critical industrial applications is still an open research problem. This paper progresses in this direction with the proposal of a novel link scheduling scheme that exploits the advantages of the schemes reported in [15] and [16] while avoiding their drawbacks. The proposed scheme is based on a centralized network management, and is hence particularly suited for industrial wireless sensor networks such as WirelessHART. The proposed scheme centrally assigns shared links to nodes that are part of the same path. However, the decision on which nodes should use a shared link at each point in time is distributed. In particular, the scheme proposes that transmitted messages act as virtual tokens to identify which node should utilize each shared link at each point in time based on the quality of previous transmissions. This hybrid (centralized and decentralized) approach provides the capacity to adapt the scheduling and utilization of links to the actual link quality conditions. This allows reducing the latency while maintaining the high reliability levels required by industrial applications. As it will be later shown, it also results in a more efficient use of resources (links and energy).

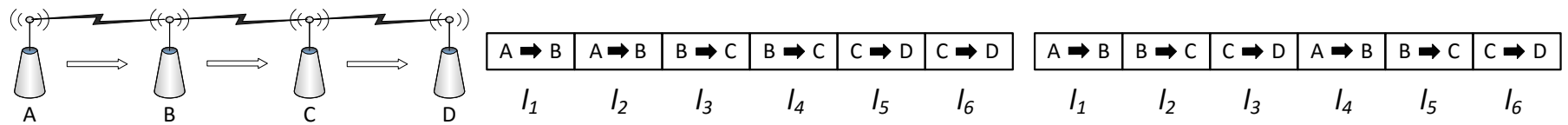

a) Path with $H=3$

b) HbH link scheduling scheme [15]

c) RTE link scheduling scheme [16]

Fig. 1. Centralized scheduling schemes

\section{Proposed Link Scheduling Scheme}

The proposed link scheduling scheme assigns links with certain flexibility in order to reduce the latency and increase the reliability in a path or multi-hop route. To this aim, the scheme proposes the use of shared links and the use of messages as virtual tokens. The scheme is referred to as LIKUID (LInK schedUlIng scheme with shareD links and virtual tokens). To explain its operation, let's consider the scenario illustrated in Fig. 1a. and define $R$ as the total number of message retransmissions allowed for the complete path by the proposed scheme. At the start of a transmission, all nodes in a path are in reception mode except for the source node ${ }^{1}$. The nodes should remain in reception mode for the following $R$ links until they correctly receive the

\footnotetext{
${ }^{1}$ Each node gets into reception mode in a different link. For example, node B gets into reception mode in $l_{1}$, while C and D do it in $l_{2}$ and $l_{3}$ respectively.
} 
message that needs to be routed to the destination node. When a node correctly receives the message, it transmits it to the next node in the multi-hop route. The source node is assigned $R+1$ links to transmit the message, and the destination node $R+1$ links to receive the message. All relay nodes in a path (i.e. nodes B and C in Fig. 1a) are assigned $R+2$ links to receive and transmit the message as illustrated in Fig. 2. A relay node cannot utilize any of the assigned links for transmission until it has correctly received the message from the predecessor node. Each hop in the path shares $R$ links with its predecessor hop. Fig. 2 shows how LIKUID assigns the different links for the scenario of Fig. 1a with $H=3$ hops in the path and a total number of message retransmissions allowed for the complete path $(R)$ equal to 1,2 and 3. The figure shows that LIKUID assigns for the complete path 4, 5 or 6 links in total when $R$ is equal to 1,2 and 3 respectively. If $R=H$, LIKUID utilizes the same number of links than $\mathrm{HbH}$ and RTE. However, if $R<H$, LIKUID assigns and utilizes in total less links than HbH and RTE. LIKUID differs from these two schemes in that all links (except the first and the last ones) are shared by various nodes; e.g. $l_{3}$ is shared for the potential transmission of the message by nodes $\mathrm{A}$, B or $\mathrm{C}$ when $R>1$. However, a single node will be able to transmit at a given point in time the message over a shared link, and the message acts as a virtual token to identify which node can transmit over the shared link. To illustrate this concept, let's consider an example when $R$ is equal to 3. Let's suppose that the first transmission of the message from node A to node $\mathrm{B}$ using $l_{1}$ is erroneous. In this case, $l_{2}$ is also utilized for the transmission of the message from node A to node B (i.e. node B remains in reception mode and does not utilize the link for the transmission to node $\mathrm{C}$ ). If the second transmission is correct, node $\mathrm{B}$ uses $l_{3}$ to transmit the message to node $\mathrm{C}$ while nodes $\mathrm{C}$ and $\mathrm{D}$ are in reception mode ${ }^{2}$. The process continues until the message is received by the destination node or the total number of message retransmissions allowed for the complete path $(R)$ is reached.

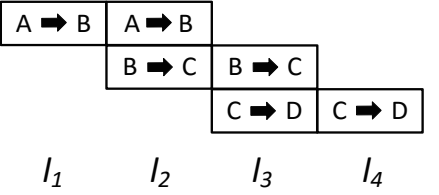

a) $R=1$

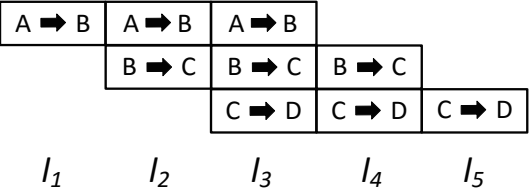

b) $R=2$

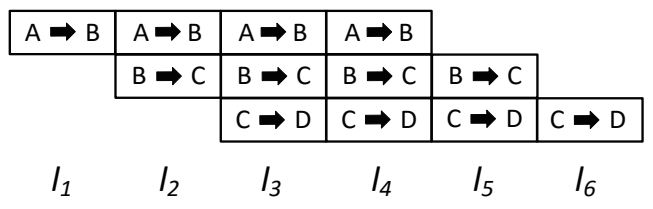

c) $R=3$

Fig. 2. LIKUID's link scheduling for a path with 3 hops when $R$ is equal to 1,2 and 3.

LIKUID can ensure the reliable delivery of a message to a destination node as long as the sum of transmission errors in all hops of the path is equal or smaller than the total number of message retransmissions allowed for the complete path $(R)$. This property is independent of the specific hops in which the transmission errors take place. LIKUID provides each node with up to $R+1$ links to guarantee the reliable delivery of a message and ensures the absence of packet collisions. In addition, LIKUID can deliver a message to the destination node in the time equivalent to $H$ links if no transmission errors occur ( $H$ is the number of hops in the path). The same latency is ensured in an error-free environment by RTE. However, this scheme can only ensure the correct delivery of the message to the destination node if the total number of transmission errors is smaller than the number of links assigned for transmission to each node. $\mathrm{HbH}$ requires more time to deliver a message to the destination in the absence of transmission errors than LIKUID and RTE. In addition, $\mathrm{HbH}$ can only ensure the correct delivery of a message to the destination node if the number of transmission errors per hop is smaller than the total number of links (including retransmissions) assigned to each hop for the transmission of a message. LIKUID can also minimize the time to deliver a message to the destination node under the presence of transmission errors. This is the case because it does not delay retransmissions, and these can take place (using the message as virtual token) as soon as the transmission error is detected. RTE cannot minimize the time to deliver a message since retransmissions need to wait for a total of $H$ links. LIKUID also maximizes the reliability for a given number of links $(H+R)$ assigned to a path or multi-hop route. This is the case because LIKUID can deliver the message to the destination node as long as the number of transmission errors is equal or smaller than $R$ ( $R$ is equal for LIKUID to the total number of retransmissions allowed for the complete path). If there are $R+1$ errors or more, no scheme with $H+R$ links could deliver the message to the destination since it will need $H+R+1$ transmissions.

The proposed scheme can be used in existing wireless industrial standards, in particular in WirelessHART and ISA100.11a. The scheme satisfies the WirelessHART requirements and should be utilized for the main path between source and destination nodes. This is the case because the WirelessHART standard only requires assigning links for possible retransmissions to the main path (such assignment is not mandatory for alternative paths). In this context, this study focuses on such main path and does not consider alternative paths. Such alternative paths would operate identically for all the schemes under study, and therefore the consideration or not of alternative paths does not affect the outcome of the present comparison study.

\footnotetext{
${ }^{2}$ If the second transmission would have been erroneous, node A will have tried for the third time the transmission of the message to node B using $l_{3}$. If this transmission would have been correct, then node B would have transmitted the message to node C using $l_{4}$.
} 


\section{Metrics}

The performance and efficiency of the different link scheduling schemes is evaluated using different metrics. Their reliability is measured using the end-to-end Packet Delivery Ratio $\left(P D R_{E 2 E}\right)$. This metric is computed as the ratio between the messages correctly received at the destination node and the total number of messages transmitted by the source node.

The end-to-end delivery delay is another critical performance metric in industrial wireless sensor networks. This metric $\left(D_{E 2 E}\right)$ is here estimated as the average number of links elapsed between the transmission of a message by the source node and its reception at the destination node. Without loss of generality, this study considers that all links utilized for the transmission of a message are allocated consecutively in a data superframe following the scheduling process defined by each scheme. The $D_{E 2 E}$ metric is computed considering only the messages that are correctly received at the destination node.

This study defines several metrics to analyze how efficiently each scheduling scheme utilizes the available links. The first one is the number of total links that are assigned by each scheme for the complete path or multi-hop route. The more efficient schemes will be those that achieve a given reliability level assigning a smaller total number of links for the complete path. The second efficiency metric is the average percentage of links that have been utilized. This metric is estimated as the ratio between the number of links utilized for the transmission of a message in a multi-hop route and the total number of links assigned for such route. It is important noting that certain scheduling schemes can result in that many links are actually not utilized even when the message cannot reach the destination node. This depends on how links have been assigned and on the distribution of transmission errors in the route. The third efficiency metric is the number of blocked links defined as the number of links during which a node has to be in transmission or reception mode for each message that is transmitted from source to destination. It is important noting that a node can only simultaneously utilize (either for transmission or reception) a single link. As a result, when a link is assigned to a given node, the node cannot utilize other links for other functions (e.g. transmitting a message to a different node). The fourth metric is the number of Unnecessary Reception Attempts (URA). This metric computes the number of times that a node was unnecessarily in reception mode for each message successfully delivered to the destination node. This can occur for example if a message that is being transmitted is not addressed to the node that is in reception mode, or simply because no message is being transmitted in the link during which the node is in reception mode. These circumstances clearly represent inefficiencies as the node could have been in sleep mode or undertaking other more productive tasks.

Finally, this study also evaluates the energy consumption of the different link scheduling schemes. The energy consumption is here estimated as the average energy consumed per message correctly received at the destination node $\left(E_{P M D}\right)$. This metric has been selected instead of the total energy consumption in order to reduce the influence of the $P D R_{E 2 E}$ on the analysis of the energy efficiency ${ }^{3}$.

\section{Analytical Performance Modeling}

This section presents analytical performance models for the different link scheduling schemes. In particular, it presents analytical expressions of the reliability and latency performance. These expressions have been obtained for a path or multi-hop route with $H$ hops between the source and destination nodes. The expressions have been derived for HbH and RTE considering that two links are assigned per hop. The expressions for LIKUID have been derived when $R$ is set equal to 1,2 and 3 .

\subsection{Reliability}

Let's denote $P D R_{i}$ as the PDR estimated for hop $i$. If we consider a stationary PDR at each hop, $P D R_{E 2 E}$ can be estimated analytically.

$\mathrm{HbH}$ assigns all the links to a given hop (including links for retransmissions) before the links for the following hop. The probability $P_{i}$ to correctly transmit a message in hop $i$ (whether using the first assigned link to this hop or the link assigned for retransmissions) can be expressed as:

$$
P_{i}=1-\left(1-P D R_{i}\right)^{L_{i}}
$$

where $L_{i}$ represents the number of links assigned to hop $i$ (two links per hop are considered in this study). Let's consider that the $P D R_{i}$ experienced at each hop of the path is independent of each other. In this case, it is possible to estimate $P D R_{E 2 E}$ for $\mathrm{HbH}$ using (1):

$$
\left.P D R_{E 2 E}\right|_{H b H}=\prod_{i=1}^{H} P_{i}=\prod_{i=1}^{H}\left(1-\left(1-P D R_{i}\right)^{2}\right)
$$

\footnotetext{
${ }^{3}$ For example, if a scheme delivers very few messages to the destination node, it will consume less energy than a scheme that delivers most of the messages to the destination since fewer transmissions will take place.
} 
RTE assigns first the links for the initial transmission of a message at each hop, and then the links for the potential retransmissions. Let's define $P_{0-r t x}$ as the probability that the message reaches the destination node without requiring any retransmissions in the path from source to destination. $P_{0-r t x}$ can be expressed as:

$$
P_{0-r t x}=\prod_{i=1}^{H} P D R_{i}
$$

Let's define $P_{r t x-h}$ as the probability that the message reaches the destination node following a retransmission of the message in hop $h . P_{r t x-h}$ can be expressed as:

$$
P_{r t x-h}=\left(1-P D R_{h}\right) \cdot \prod_{i=1}^{H} P D R_{i}
$$

where $\left(1-P D R_{h}\right)$ represents the probability that the message is first not correctly transmitted in hop $h$ and a retransmission is necessary. We can compute the probability $P_{1-r t x}$ that the message is correctly received at the destination node after a single retransmission at any of the $H$ hops of the path. This probability is equal to the sum of the $P_{r t x-h}$ probabilities for all the $H$ hops in the path:

$$
P_{1-r t x}=\sum_{h=1}^{H} P_{r t x-h}=\left(\prod_{i=1}^{H} P D R_{i}\right) \cdot\left(\sum_{i=1}^{H}\left(1-P D R_{i}\right)\right)
$$

$P D R_{E 2 E}$ can then be estimated for RTE as ${ }^{4}$ :

$$
\left.P D R_{E 2 E}\right|_{R T E}=P_{0-r t x}+P_{1-r t x}=\left(\prod_{i=1}^{H} P D R_{i}\right)+\left(\prod_{i=1}^{H} P D R_{i}\right) \cdot\left(\sum_{i=1}^{H}\left(1-P D R_{i}\right)\right)=\left(\prod_{i=1}^{H} P D R_{i}\right) \cdot\left(1+\sum_{i=1}^{H}\left(1-P D R_{i}\right)\right)
$$

It is possible to obtain for LIKUID an analytical expression of $P D R_{E 2 E}$ that is a function of $R$ (total number of message retransmissions allowed for the complete path). When $R$ is set equal to one (i.e. only one retransmission is allowed), the message is delivered to the destination node if there are no transmission errors $\left(P_{0-r t x}\right.$ in eq. (3)) or there is only one transmission error $\left(P_{1 \text { - }}\right.$ ${ }_{r t x}$ in eq. (5)). The $P D R_{E 2 E}$ of LIKUID when $R$ is equal to one is then the sum of $P_{0-r t x}$ and $P_{1-r t x}$, and its value is represented by eq. (6). When $R$ is set equal to 2, the message can also be delivered to the destination node if there are two retransmissions in total (independently of the hop in which they take place). Following the same process used to define $P_{1 \text {-rtx }}$, we can compute the probability $P_{2-r t x}$ that the message is correctly received at the destination node after two retransmissions:

$$
P_{2-r t x}=\left(\prod_{i=1}^{H} P D R_{i}\right) \cdot\left(\sum_{i=1}^{H} \sum_{j=i}^{H}\left(1-P D R_{i}\right) \cdot\left(1-P D R_{j}\right)\right)
$$

Following the same process, we can also estimate $P_{3-r t x}$ :

$$
P_{3-r t x}=\left(\prod_{i=1}^{H} P D R_{i}\right) \cdot\left(\sum_{i=1}^{H} \sum_{j=i}^{H} \sum_{k=j}^{H}\left(1-P D R_{i}\right) \cdot\left(1-P D R_{j}\right) \cdot\left(1-P D R_{k}\right)\right)
$$

Finally, the $P D R_{E 2 E}$ for LIKUID can then be expressed as function of $R$ following eq. (9):

$$
\left.P D R_{E 2 E}\right|_{\text {LIKUID }}=\left\{\begin{array}{rr}
P_{0-r t x}+P_{1-r t x}=\left(\prod_{i=1}^{H} P D R_{i}\right) \cdot\left(1+\sum_{i=1}^{H}\left(1-P D R_{i}\right)\right) & \text { if } \\
P_{0-r t x}+P_{1-r t x}+P_{2-r t x}=\left(\prod_{i=1}^{H} P D R_{i}\right) \cdot\left(1+\left(\sum_{i=1}^{H}\left(1-P D R_{i}\right)\right)+\left(\sum_{i=1}^{H} \sum_{j=i}^{H}\left(1-P D R_{i}\right) \cdot\left(1-P D R_{j}\right)\right)\right) & \text { if } \quad R=2 \\
P_{0-r t x}+P_{1-r t x}+P_{2-r t x}+P_{3-r t x}=\left(\prod_{i=1}^{H} P D R_{i}\right) \cdot\left(\begin{array}{l}
\left.1+\left(\sum_{i=1}^{H}\left(1-P D R_{i}\right)\right)+\left(\sum_{i=1}^{H} \sum_{j=i}^{H}\left(1-P D R_{i}\right) \cdot\left(1-P D R_{j}\right)\right)\right) \\
+\left(\sum_{i=1}^{H} \sum_{j=i}^{H} \sum_{k=j}^{H}\left(1-P D R_{i}\right) \cdot\left(1-P D R_{j}\right) \cdot\left(1-P D R_{k}\right)\right)
\end{array}\right) & \text { if } \quad R=3
\end{array}\right.
$$

\footnotetext{
${ }^{4}$ It is important to remember that when RTE is configured with two links per hop, it will not be able to deliver the message to the destination node if there are more than two transmission errors.
} 


\subsection{Latency}

If we consider again that the PDR experienced at each hop $i\left(P D R_{i}\right)$ is stationary, it is possible to derive $D_{E 2 E}$ analytically as a function of the number $H$ of hops between the source and destination nodes.

$\mathrm{HbH}$ assigns consecutively all the links to be utilized at each hop of a path. The links assigned to the final hop are then placed at the end. The $D_{E 2 E}$ metric can be computed for $\mathrm{HbH}$ as the sum of two delay components $D_{0}$ and $D_{1} . D_{0}$ is the end-to-end delay experienced when the message reaches the destination node without needing a retransmission at the last hop. $D_{1}$ is the end-to-end delay experienced when the message reaches the destination node after a retransmission at the last hop. $D_{0}$ is obtained by multiplying the probability that the message reaches the last relay node by the probability that the message correctly reaches the destination node without needing a retransmission at the last hop $\left(P D R_{H}\right)$ and by the corresponding delay $(2(H-1)+1)$. The result of this multiplication is then divided by $P D R_{E 2 E}$ to obtain $D_{0} . D_{1}$ is obtained multiplying the probability that the message reaches the last relay node by the probability that the message correctly reaches the destination node after a retransmission at the last hop $\left(P D R_{H}\right)$ and by the corresponding delay $2 H$. The result of this multiplication is then divided by $P D R_{E 2 E}$ to obtain $D 1$. The $D_{E 2 E}$ metric can then be expressed as:

$$
\left.D_{E 2 E}\right|_{H b H}=D_{0}+D_{1}=\frac{\left(\prod_{i=1}^{H-1}\left(1-\left(1-P D R_{i}\right)^{2}\right)\right) \cdot P D R_{H} \cdot(2 \cdot(H-1)+1)}{\prod_{i=1}^{H}\left(1-\left(1-P D R_{i}\right)^{2}\right)}+\frac{\left(\prod_{i=1}^{H-1}\left(1-\left(1-P D R_{i}\right)^{2}\right)\right) \cdot\left(1-P D R_{H}\right) \cdot P D R_{H} \cdot(2 \cdot H)}{\prod_{i=1}^{H}\left(1-\left(1-P D R_{i}\right)^{2}\right)}
$$

It is possible to simplify eq.(10) and obtain a $D_{E 2 E}$ metric for $\mathrm{HbH}$ that only depends on $P D R_{H}$ and $H$ :

$$
\left.D_{E 2 E}\right|_{H b H}=\frac{P D R_{H} \cdot(2 \cdot(H-1)+1)}{\left(1-\left(1-P D R_{H}\right)^{2}\right)}+\frac{\left(1-P D R_{H}\right) \cdot P D R_{H} \cdot(2 \cdot H)}{\left(1-\left(1-P D R_{H}\right)^{2}\right)}=\frac{P D R_{H} \cdot\left((2 \cdot(H-1)+1)+\left(1-P D R_{H}\right) \cdot(2 \cdot H)\right)}{\left(1-\left(1-P D R_{H}\right)^{2}\right)}
$$

The $D_{E 2 E}$ metric for RTE can be expressed as follows:

$$
\left.D_{E 2 E}\right|_{R T E}=\frac{P_{0-r \times x} \cdot H}{\left.P D R_{E 2 E}\right|_{R T E}}+\frac{P_{1-r x x} \cdot 2 \cdot H}{\left.P D R_{E 2 E}\right|_{R T E}}=\frac{\left(\prod_{i=1}^{H} P D R_{i}\right) \cdot H+\left(\prod_{i=1}^{H} P D R_{i}\right) \cdot\left(\sum_{i=1}^{H}\left(1-P D R_{i}\right)\right) \cdot(2 \cdot H)}{\left(\prod_{i=1}^{H} P D R_{i}\right) \cdot\left(1+\left(\sum_{i=1}^{H}\left(1-P D R_{i}\right)\right)\right)}=\frac{\left(H+(2 \cdot H) \cdot\left(\sum_{i=1}^{H}\left(1-P D R_{i}\right)\right)\right)}{\left(1+\left(\sum_{i=1}^{H}\left(1-P D R_{i}\right)\right)\right)}
$$

The first term in eq. (12) is equal to the probability $P_{0-r t x}$ (eq. (3)) multiplied by the related delay $(H)$ and divided by $P D R_{E 2 E}$. The second term is equal to the probability $P_{1-r t x}$ (eq. (5)) multiplied by the corresponding delay $(2 \cdot H)$ and divided by $P D R_{E 2 E}$.

The $D_{E 2 E}$ metric for LIKUID can be obtained as the sum of $R+1$ terms:

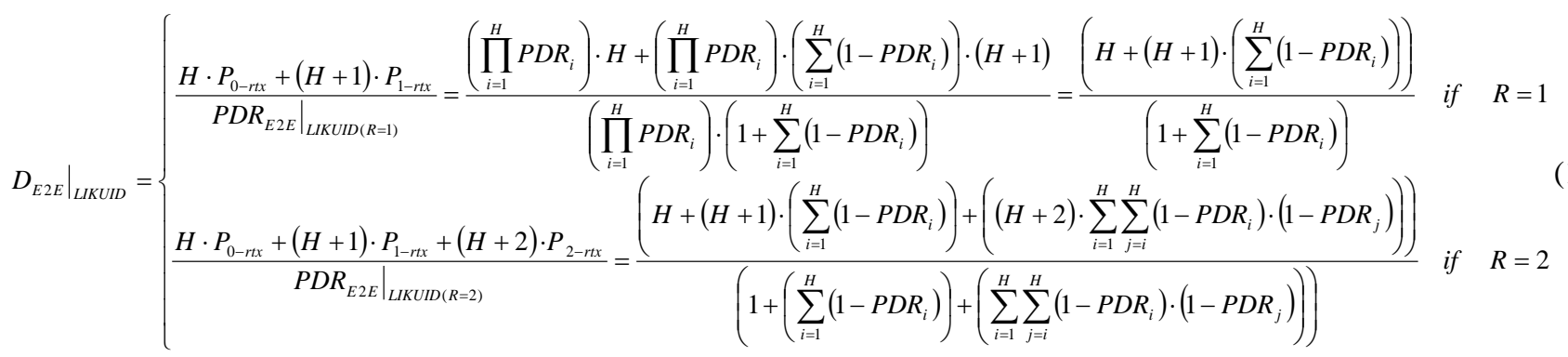

When $R$ is equal to 1 , the first term in eq. (13) results from the multiplication of the probability $P_{0-r t x}$ (eq. (3)) by the corresponding delay $(H)$ divided by $P D R_{E 2 E}$. The second term in eq. (13) results from the multiplication of the probability $P_{1-r t x}$ (eq. (5)) by the corresponding delay $(H+1)$ divided by $P D R_{E 2 E}$. When $R$ is equal to $2, D_{E 2 E}$ is obtained summing a third term that is the result of multiplying the probability $P_{2-r t x}$ by its corresponding delay $(H+2)$ divided by $P D R_{E 2 E}$. The same process is followed to obtain $D_{E 2 E}$ for higher values of $R^{5}$.

\section{Performance evaluation}

The performance of the proposed link scheduling scheme is compared against that obtained with $\mathrm{HbH}$ and RTE. We also consider a fourth simple scheduling scheme that does not consider retransmissions. This scheme, referred to as NoRtx, only needs to assign one link to each hop in a path. Its evaluation is here included to highlight the impact of retransmissions.

\footnotetext{
${ }^{5}$ When $R$ is equal to 3, we will need to add an additional term that results from the multiplication of $P_{3-r t x}$ (eq. (8)) by its corresponding delay ( $\left.H+3\right)$ divided by $P D R_{E 2 E}$. The $D_{E 2 E}$ expression is not shown here due to its complexity.
} 
All schemes are analyzed under the four multi-hop scenarios depicted in Fig. 3. The four scenarios represent the deployment of nodes along a typical corridor that is characteristic of factories. The reliability and latency performance of the scheduling schemes has been numerically evaluated in scenarios 1 and 2 using the analytical expressions presented in the previous section. On the other hand, the efficiency and energy are obtained through simulations using the Monte Carlo method. The schemes are evaluated (for all the metrics) through simulations in scenarios 3 and 4. To ensure the statistical accuracy of the obtained results, sufficient numerical and simulation runs have been executed in order to achieve a relative error below $1 \%$ for all results in the four scenarios.

The first scenario considers a path or multi-hop route with $H=3$ and the same PDR experienced in each hop (i.e. $\left.P D R_{A B}=P D R_{B C}=P D R_{C D}\right)$. The first scenario has been selected to analyze the performance of the proposed schemes under different link quality levels by changing the PDR experienced at each hop. The second scenario extends the number of hops to four, and also considers the same PDR per hop $\left(P D R_{A B}=P D R_{B C}=P D R_{C D}=P D R_{D E}\right)$. The second scenario has been implemented to investigate the effect of the number hops between source and destination on the performance of the link scheduling schemes. It is important noting that four hops have been selected between source and destination since this is the maximum number of hops recommended by the HART Communication Foundation for a multi-hop industrial wireless link. The third scenario is used to evaluate the performance of the link scheduling schemes using simulations that represent the link level fluctuations experienced in industrial wireless communications. Such fluctuations are reproduced using an empirically derived radio propagation model for industrial environments. Two distances between nodes (50 and 150 meters) have been simulated to account for different average link quality levels. The performance of the different schemes has then been evaluated for all possible eight combinations of distances between nodes for the 3-hop scenario depicted in Fig. 3. The fourth scenario considers a mobile node A that moves within the radio coverage of node $\mathrm{B}^{6}$ following the random waypoint mobility model [17]. This scenario considers that node $\mathrm{A}$ always uses node $\mathrm{B}$ to transmit messages to the destination node $\mathrm{D}$. In this case, it is not necessary to reprogram the assignment of links as a result of the mobility of node A. In this fourth scenario, the maximum distance between nodes A and B ( $\left.d_{A B}\right)$ is set equal to 150 meters. Two values (50m and 150m) have also been considered for the distance between static nodes. The fourth scenario has been implemented to evaluate the link scheduling schemes under variable link quality conditions resulting from the mobility of a node.

For the simulations, all functions that intervene in the link scheduling process have been implemented in Matlab. In particular, the implemented simulation platform includes a radio propagation model for industrial wireless communications, the data link layer, the nodes' mobility and the different link scheduling schemes evaluated. This study assumes that all nodes have been informed by the Network Manager of the route they should use to transmit their data to the destination node.

The schemes have been evaluated considering that the nodes integrate the XDM2510H WirelessHART radio transceiver [18]. The transceiver is powered with $3.6 \mathrm{~V}$, and has a power consumption of $18 \mathrm{~mA}$ and $6 \mathrm{~mA}$ when it is in transmission and reception mode, respectively. Its transmission power is $8 \mathrm{dBm}$ and its receiver sensibility is equal to $-90 \mathrm{dBm}$. The energy consumption is computed considering that nodes transmit messages of 133 Bytes, and that the correct or incorrect reception of a message in each hop is acknowledged with a message of 26 Bytes.

Radio propagation conditions in scenarios 3 and 4 have been simulated using the model presented in [19] for industrial environments. The model considers one-slope path-loss, and models the fading effects at 2.4GHz. Some of the model parameters are: $d_{0}=15 \mathrm{~m}$ (reference distance), $P L\left(d_{0}\right)=71.84 \mathrm{dBm}$ (path-loss at $d_{0}$ distance), $n=2.16$ (path-loss exponent), $\sigma=8.13 \mathrm{dBm}$ (shadowing standard deviation) and $G t=G r=0 \mathrm{~dB}$ (transmitter and receiver antenna gains). In this study, we consider that a message is correctly received if the RSSI (Received Signal Strength Indicator) is above -90dBm. It is important noting that, as required by WirelessHART, no packet collisions can occur with the four scheduling schemes under evaluation.
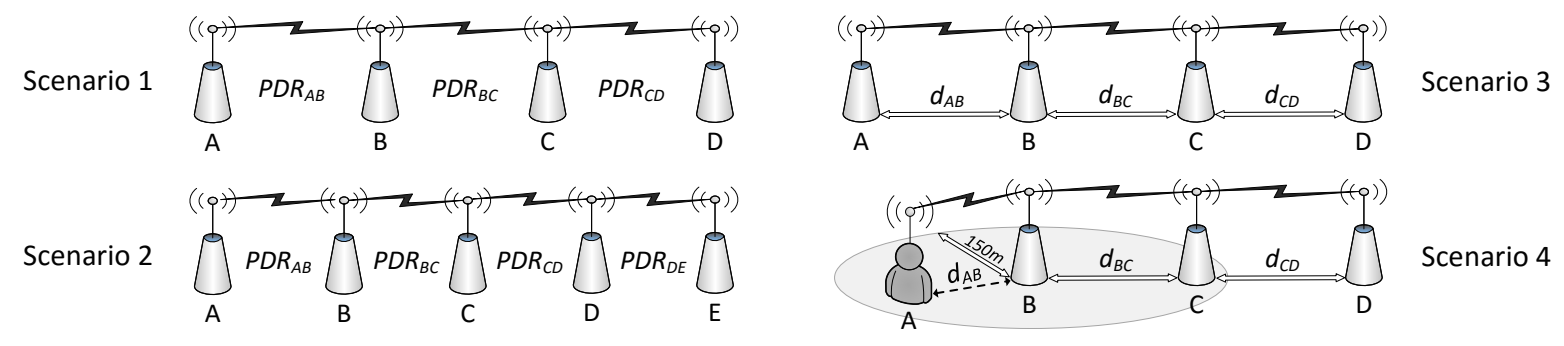

Fig. 3. Evaluation scenarios.

\footnotetext{
${ }^{6}$ This restriction aims to represent the fact that in factories, the mobility of nodes is generally limited to their working environment, e.g. workers usually move around the machinery they manipulate.
} 


\subsection{Validation of analytical expressions}

The reliability and latency evaluation under scenarios 1 and 2 are conducted using the analytical expressions presented in Section 5. It is then important to validate these expressions by comparing the results obtained when using the analytical expressions of $P D R_{E 2 E}$ and $D_{E 2 E}$ and when estimating these metrics through simulations. Fig. 4 depicts such comparison ${ }^{7}$ when considering the first scenario represented in Fig. 3 and $R$ is set equal to 1, 2, 3 and 4 (in this case, we refer to LIKUID as LIKUID ${ }_{1}$, LIKUID LIKUID $_{3}$, and LIKUID 4 respectively). The figure shows for all schemes a close match between the results obtained using the analytical expressions (Ana) and using simulations (Sim). These results validate the derived analytical expressions.

\subsection{Scenario 1}

Fig. 4a depicts the probability that a message is correctly received by the destination node $\left(P D R_{E 2 E}\right)$ as a function of the PDR experienced at each hop $\left(P D R_{i}\right)$. These results have been obtained for the first evaluation scenario (Fig. 3$)$ that considers a path or multi-hop route with $H=3$ and $P D R_{A B}=P D R_{B C}=P D R_{C D}$. Results for LIKUID are shown for $R=1\left(\operatorname{LIKUID}_{1}\right), R=2\left(\mathrm{LIKUID}_{2}\right)$ and $R=3\left(\mathrm{LIKUID}_{3}\right)$. It is important remembering that the configuration with $R=3$ corresponds to the case in which LIKUID utilizes the same total number of links as $\mathrm{HbH}$ and RTE in scenario 1 (Table 1). For a path with 3 hops, this total number of links is equal to six (Fig. 2), which is also the minimum number of links necessary for $\mathrm{HbH}$ and RTE to allow for possible retransmissions at every hop of the path. LIKUID can allow retransmissions at any hop of the path using a lower total number of links for the complete path. Fig. 2 showed that, for example, when LIKUID is configured with $R=1$, the total number of links assigned for the path or multi-hop route is equal to four (Table 1). In this case, only one retransmission is allowed for the complete path, but such retransmission can take place at any hop of the path. The results depicted in Fig. 4a show that LIKUID $_{2}$ and $\mathrm{LIKUID}_{3}$ outperform the other schemes for all possible values of $P D R_{i}$. This means that LIKUID can achieve higher $P D R_{E 2 E}$ than $\mathrm{HbH}$ and RTE even if it assigns one link less (i.e. $R=2$ ) for the complete path (Table 1). The obtained results also show that decreasing $P D R_{i}$ values have a smaller effect on LIKUID than on the other schemes. For example, when $P D R_{i}$ is equal to 0.95 , LIKUID $_{3}$ achieves a $P D R_{E 2 E}$ equal to 0.9999 , and $\mathrm{HbH}$ and RTE a $P D R_{E 2 E}$ equal to 0.9925 and 0.9860 respectively. When the $P D R_{i}$ decreases to $0.75, \mathrm{LIKUID}_{3}$ can maintain a high $P D R_{E 2 E}(0.9624)$ while the $P D R_{E 2 E}$ for $\mathrm{HbH}$ and RTE is significantly degraded to 0.8240 and 0.7383 respectively. Fig. 4a also shows that $\mathrm{HbH}$ achieves a higher $P D R_{E 2 E}$ than RTE. This is the case because $\mathrm{HbH}$ allows for a possible retransmission at every hop of the path, while RTE only allows for one retransmission in the complete path. $\operatorname{LIKUID}_{1}(R=1)$ also allows for only one retransmission in the complete path. However, it only assigns 4 links compared to the 6 links assigned by RTE (Table 1). The poor reliability performance achieved by NoRtx emphasizes the importance of retransmissions to ensure high $P D R_{E 2 E}$ results.

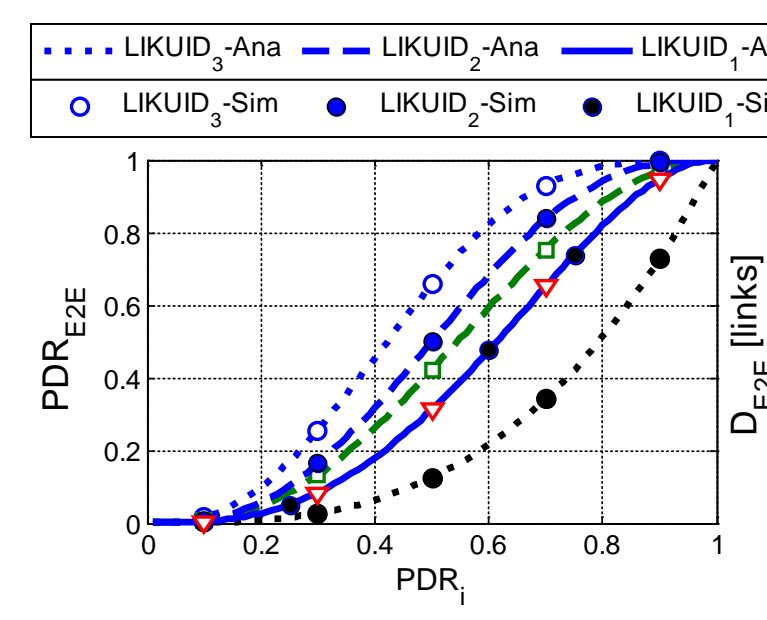

(a) Reliability

\begin{tabular}{|c|c|c|c|c|}
\hline -Ana & $--\mathrm{HbH}-\mathrm{Ana}$ & - - = RTE-Ana & $\cdot-$ & NoRtx-Ana \\
\hline Sim & $\mathrm{HbH}-\mathrm{Sim}$ & RTEe-Sim & - & NoRtx-Sim \\
\hline
\end{tabular}

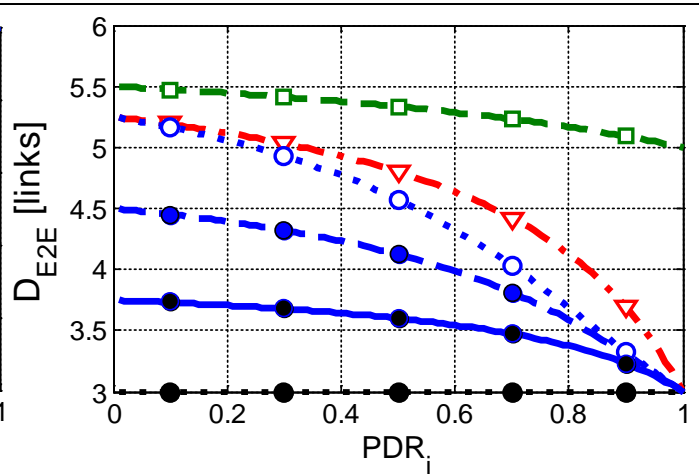

(b) Latency

Fig. 4. Reliability and latency as a function of the $P D R_{i}$ for scenario 1.

Table 1 - Number of total assigned links as a function of the number of hops in the path

\begin{tabular}{|c|c|c|c|c|c|c|c|}
\hline$H$ & LIKUID $_{4}$ & $\mathrm{LIKUID}_{3}$ & ${ }_{3}$ LIKUID $_{2}$ & LIKUID $_{1}$ & $\mathrm{HbH}$ & RTE & NoRtx \\
\hline 3 & - & 6 & 5 & 4 & 6 & 6 & 3 \\
\hline 4 & 8 & 7 & 6 & 5 & 8 & 8 & 4 \\
\hline
\end{tabular}

\footnotetext{
${ }^{7}$ The results are obtained considering the same $P D R_{i}$ value for each hop in the path..
} 
Fig. 4b represents the end-to-end delivery delay $\left(D_{E 2 E}\right)$ that is estimated as the average number of links elapsed between the transmission of a message by the source node and its reception at the destination node. It is important remembering that $D_{E 2 E}$ is computed considering only the messages correctly received by the destination node. As a result, the delay results have to be discussed taking into account the $P D R_{E 2 E}$ achieved by each scheme. Fig. $4 \mathrm{~b}$ shows that $\mathrm{HbH}$ experiences the higher delivery delay $D_{E 2 E}$. This is the case because $\mathrm{HbH}$ can only deliver the message to the destination node at $l_{5}$ even if there are no transmission errors, whereas all the other schemes can do so at $l_{3}$. The minimum possible delay $D_{E 2 E}$ is hence equal to 5 for $\mathrm{HbH}$, and equal to 3 for all other schemes. NoRtx has a delay always equal to 3 since retransmissions are not allowed and only received messages at the destination node are taken into account when computing $D_{E 2 E}$. However, NoRtx exhibits a poor $P D R_{E 2 E}$ performance (Fig. 4a), in particular under low $P D R_{i}$ values. As it would be expected, RTE reduces the delay compared to $\mathrm{HbH}$. However, RTE increases the delay compared to all the configurations of the LIKUID proposal, including the one that utilizes the same total number of links as RTE (i.e. LIKUID 3 ). Fig. 4 shows that LIKUID $_{1}$ can significantly reduce the delay compared to $\mathrm{PbP}$ even if both schemes achieve the same reliability levels. $\mathrm{LIKUID}_{2}$ and $\mathrm{LIKUID}_{3}$ increase the ratio of messages delivered to the destination node compared to $\mathrm{HbH}$ and RTE, and they do so while reducing the time to deliver them. These results clearly demonstrate that using shared links and virtual tokens can reduce unnecessary waiting times at intermediate hops and increase the reliability at each hop of the path. Of particular relevance are the results obtained with LIKUID $_{2}$. This configuration can increase the $P D R_{E 2 E}$ and decrease the $D_{E 2 E}$ (compared to $\mathrm{HbH}$ and RTE) while reducing the total number of links assigned to a complete path or multi-hop route (Table 1). The released link can be utilized for other purposes including augmenting the number of programmed paths or increasing their $P D R_{E 2 E}$.

Fig. 5a represents the average percentage of links that have been utilized for the transmission of a message as a function of the $P D R_{i}$. The depicted results show that LIKUID always operates more efficiently than HbB and RTE as it achieves a higher average utilization of the assigned links for all the evaluated $P D R_{i}$ values. A joint analysis of the results in Fig. 4 and Fig. 5 reveals some interesting findings. Existing schemes (HbH, RTE and NoRtx) exhibit a low utilization of links (around 33\%) when operating under low $P D R_{i}$ values (between 0 and 0.2). Such utilization level indicates that few messages are delivered to the destination node, and few actually even reach the second hop of the path. LIKUID increases significantly the utilization level under low $P D R_{i}$ values (Fig. 5a), but this does not have a significant impact on the $P D R_{E 2 E}$ (Fig. 4a). Fig. 5a also shows that LIKUID can increase the average percentage of links that have been utilized under high $P D R_{i}$ values (between 0.9 and 1 ) when using a smaller total number of links for the complete path (i.e. LIKUID ${ }_{1}$ has higher utilization level than LIKUID $_{2}$ and LIKUID $_{3}$ ). This is the case because under good radio link quality conditions, messages do not generally require retransmissions to reach the destination node. The links reserved for such retransmissions are therefore rarely utilized.

Fig. 5b depicts the number of Unnecessary Reception Attempts (URA) for each message successfully delivered to the destination as a function of the $P D R_{i}$. Previous results have clearly demonstrated the significant reliability and latency benefits of LIKUID. However, such benefits come at the expense of increasing the URA under low $P D R_{i}$ values, and in particular when utilizing the same total number of links as $\mathrm{HbH}$, RTE or NoRtx (i.e. LIKUID ${ }_{3}$ ). A higher number of retransmissions take place in the first hops of the path when experiencing low $P D R_{i}$ values. In this case, nodes close to the destination node will unnecessarily be in reception mode for a larger number of links. This drawback is mitigated as the $P D R_{i}$ augments and URA decreases. Also, the URA can be decreased for LIKUID by reducing the total number of links assigned to a complete path or multi-hop route. For example, $\mathrm{LIKUID}_{2}$ experiences the same URA levels as $\mathrm{HbH}$ while increasing $P D R_{E 2 E}$ and reducing $D_{E 2 E}$.

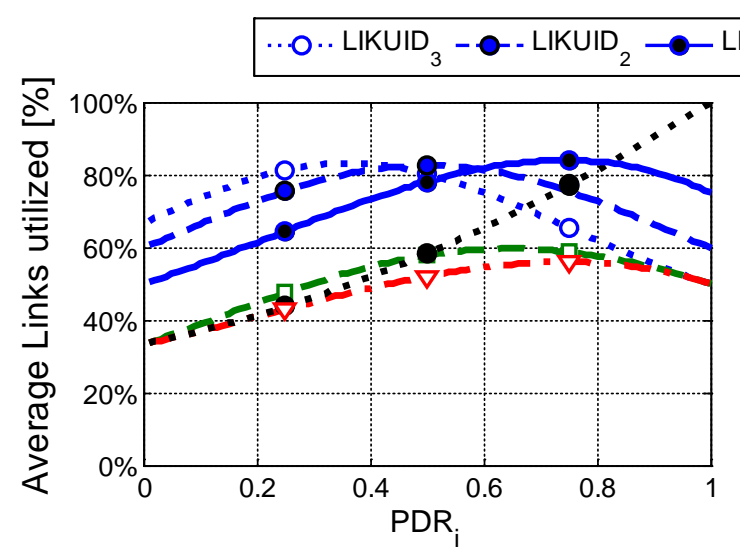

(a) Average percentage of links that have been utilized

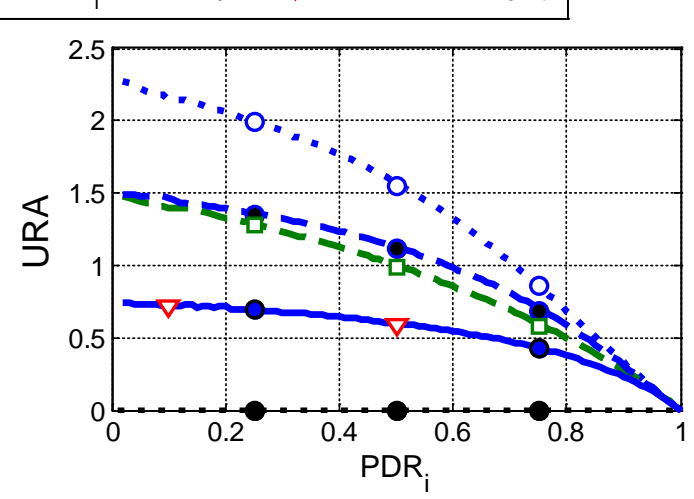

(b) URA

Fig. 5. Average percentage of links that have been utilized and Unnecessary Reception Attempts (URA) as a function of the $P D R_{i}$ for scenario 1. 
The total energy consumption $(E)$ is shown in Fig. 6a as a function of the $P D R_{i}$. The total energy consumption varies with the $P D R_{i}$, but all algorithms converge to the same energy consumption value (approximately $1180 \mu \mathrm{J}$ ) when the $P D R_{i}$ is equal to 1 .

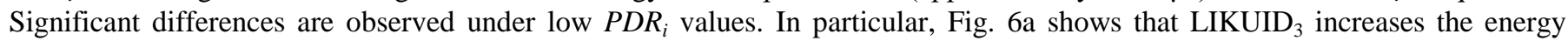
consumption. However, $\mathrm{LIKUID}_{3}$ is the scheme achieving the highest $P D R_{E 2 E}$ levels (Fig. 4a), and reliability is the most critical aspect in industrial applications. The high energy consumption levels characterizing LIKUID are due to two main factors. The first one is the fact that LIKUID achieves the highest $P D R_{E 2 E}$. The higher the packet delivery ratio, the more messages are transmitted, and therefore more energy is consumed for their transmission. The second factor is related to the higher URA values measured with LIKUID (Fig. 5b), and that result from the fact that intermediate nodes must be in reception mode in case a message is sent to them (virtual token concept). Although this has a cost in terms of URA and energy consumption, it provides LIKUID with higher flexibility. Such flexibility is at the origin of the reliability and latency gains achieved by LIKUID with respect to existing schemes. Since the different scheduling schemes result in varying $P D R_{E 2 E}$ levels, a more fair comparison of the energy performance is possible if we consider the average energy consumed per message correctly received at the destination node $\left(E_{P M D}\right)$. Fig. $6 \mathrm{~b}$ represents the $E_{P M D}$ as a function of $P D R_{i}$. As it could be expected, the higher the $P D R_{i}$ the lower the $E_{P M D}$ metric since fewer retransmissions are needed. For high $P D R_{i}$ values, few differences between the schemes can be observed. The differences appear when operating under low $P D R_{i}$ values. In this case, $\mathrm{LIKUID}_{3}$ results in the lowest $E_{P M D}$ values. This result demonstrates that although $\mathrm{LIKUID}_{3}$ increases the total energy consumption as a result of its higher $P D R_{E 2 E}$, it is more efficient than existing schemes.

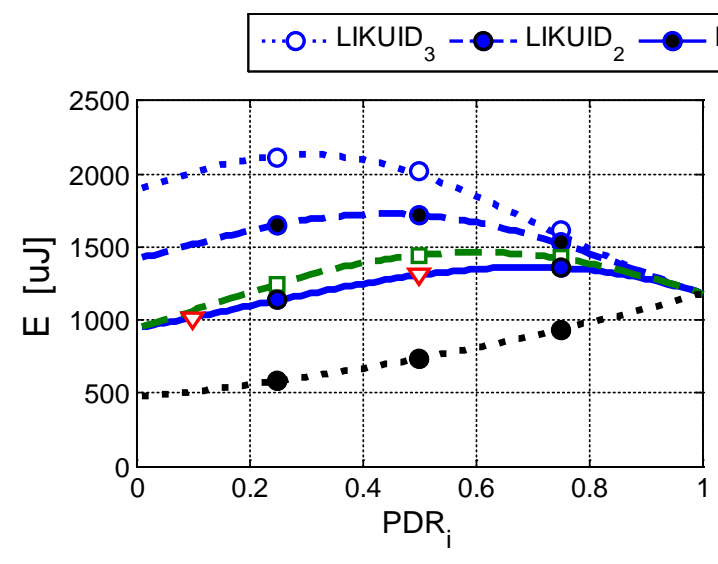

(a) Total Energy

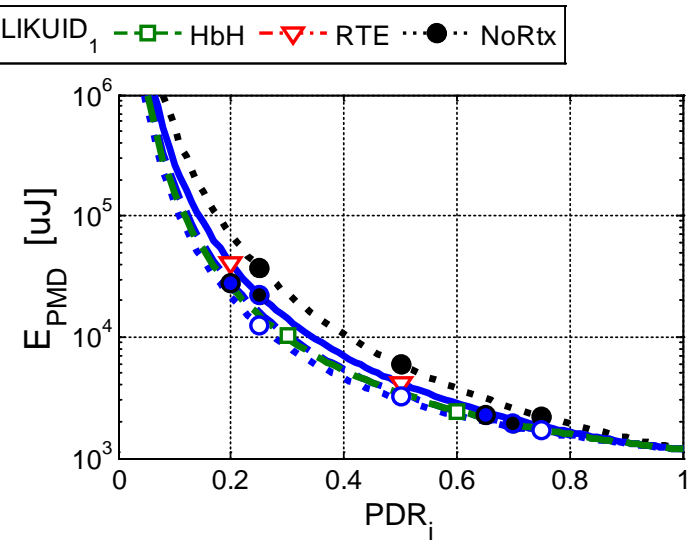

(b) Energy per message delivery

Fig. 6. Total energy consumption and average energy consumed per message correctly received at the destination node as a function of the $P D R_{i}$ for scenario 1.

Finally, Table 2 presents for the first evaluation scenario the number of blocked links defined as the number of links during which a node has to be in transmission or reception mode for each message that is transmitted from source to destination. The number of links during which a node is blocked only depends on the number of hops $H$. In this case, the results depicted in Table 2 are valid for the first, third and fourth evaluation scenarios since all of them are characterized by $H=3$. LIKUID 3 has the highest number of blocked links. LIKUID ${ }_{2}$ only results in one more blocked link at the source and destination nodes compared to $\mathrm{HbH}$ and RTE. LIKUID 1 reduces the number of blocked links compared to HbH and RTE (Table 2) and the total number of links assigned to the complete path or multi-hop route (Table 1). It is important noting that LIKUID $_{1}$ improved the $D_{E 2 E}$ performance compared to RTE (Fig. 4), and achieved the same $P D R_{E 2 E}$ as $\mathrm{HbH}$ and RTE while reducing the total number of links assigned to the complete path (Table 1).

Table 2 - Blocked links $(H=3)$

\begin{tabular}{c|cccccc}
\hline \hline Node & LIKUID $_{3}$ & LIKUID $_{2}$ & LIKUID $_{1}$ & HbH & RTE & NoRtx \\
\hline A & 4 & 3 & 2 & 2 & 2 & 1 \\
B & 5 & 4 & 3 & 4 & 4 & 2 \\
C & 5 & 4 & 3 & 4 & 4 & 2 \\
D & 4 & 3 & 2 & 2 & 2 & 1 \\
\hline \hline
\end{tabular}

\subsection{Scenario 2}

The second evaluation scenario adds an additional hop in the path with respect to the first scenario (Fig. 3). In this case, HbH and RTE assign two links per hop, and therefore a total of 8 links for the complete path (Table 1). In this scenario, LIKUID 3 only requires 7 links for the complete path. There is one link per hop, and three additional links for possible retransmissions $(R=3)$. We have also evaluated in this scenario $\mathrm{LIKUID}_{4}(R=4)$ since this configuration utilizes the same total number of links as HbH 
and RTE. In general terms, the same performance trends have been observed for scenario 2 as for scenario 1 . This section hence focuses on the impact of incrementing the number of hops in the path.

The results in Fig. 7a confirm that LIKUID outperforms $\mathrm{HbH}$ and RTE when there are at least two shared links for retransmissions and $H$ is equal or smaller than 4 (i.e. LIKUID $_{2}$, LIKUID 3 and LIKUID $_{4}$ when $\left.H=4\right)^{8}$. It is also important noting that the $P D R_{E 2 E}$ gains achieved with LIKUID with respect to the other schemes increase with the number of hops. For example, when $P D R_{i}$ is equal to $0.95, \mathrm{LIKUID}_{3}$ achieves a $P D R_{E 2 E}$ of 0.9998 under scenario 2 (similar performance to that achieved under scenario 1 when the $P D R_{E 2 E}$ was equal to 0.9999). On the other hand, $\mathrm{HbH}$ and RTE see their $P D R_{E 2 E}$ performance reduce to 0.9885 and 0.9765 in scenario 2 compared to 0.9925 and 0.9860 respectively under scenario 1 . It is also relevant noting that LIKUID 4 increases the $P D R_{E 2 E}$ to 0.99998 . In comparison, $\mathrm{LIKUID}_{3}$ achieved a $P D R_{E 2 E}$ equal to 0.9999 in scenario $1^{9}$. Fig. 7b shows that similar $D_{E 2 E}$ performance trends to those observed under scenario 1 (Fig. $4 \mathrm{~b}$ ) are again experienced under scenario 2, with LIKUID outperforming again $\mathrm{HbH}$ and RTE. The delay increases with the number of hops for all link scheduling schemes. However, LIKUID experiences a smaller increase compared to the other schemes. Fig. 7a and Fig. 7b show that even if we reduce the total number of links assigned to the path to 6 ( $\mathrm{HbH}$ and RTE require 8 links when $H=4$ ), LIKUID $_{2}$ still outperforms $\mathrm{HbH}$ and RTE both in terms of reliability and latency.

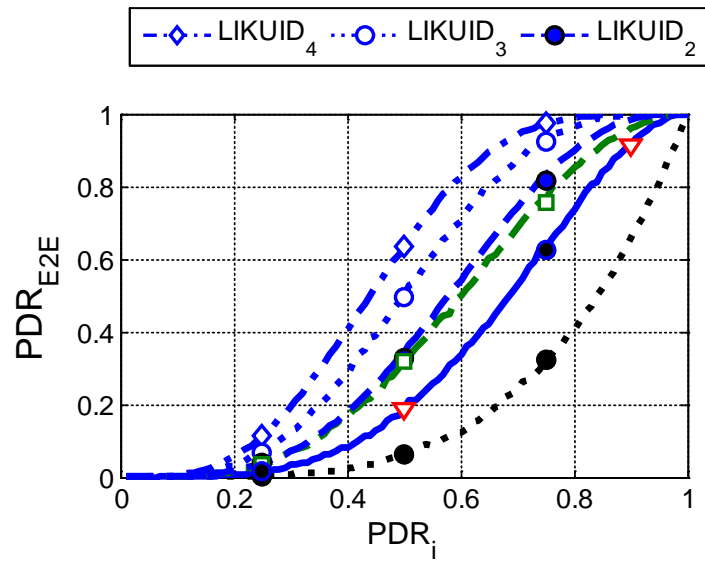

(a) Reliability
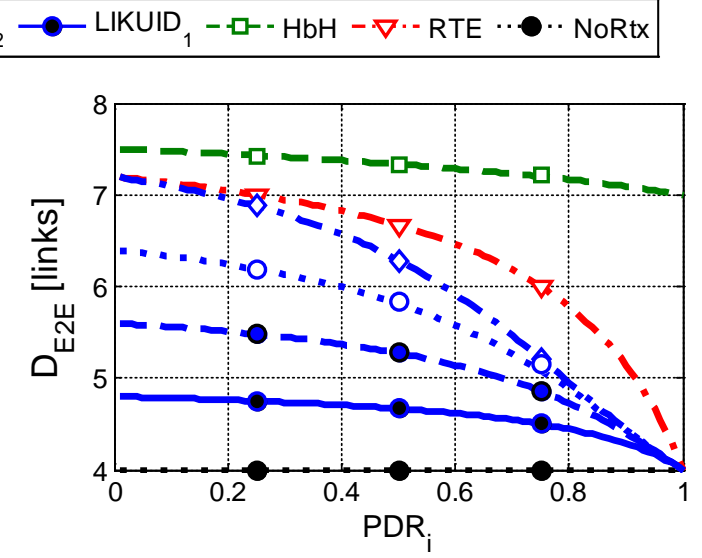

(b) Latency

Fig. 7. Reliability and latency as a function of the $P D R_{i}$ for scenario 2 .

Few differences with respect to scenario 1 where found when comparing the efficiency of the link scheduling schemes under scenario 2 (Fig. 8). Fig. 8 shows that LIKUID increases the average percentage of links that have been utilized under scenario 2 compared to scenario 1 . This is the case because there are more shared links for retransmissions. The URA metric also increases under scenario 2 since more nodes participate in the transmission of the message.

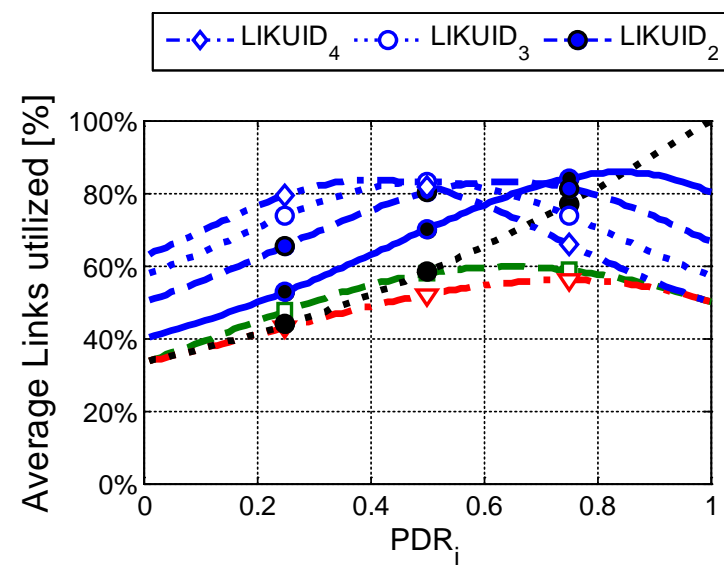

(a) Average percentage of links that have been utilized

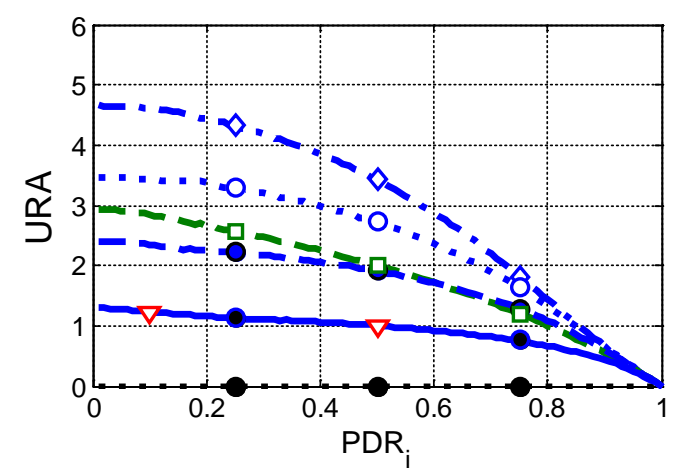

(b) URA

Fig. 8. Average percentage of links that have been utilized and Unnecessary Reception Attempts (URA) as a function of the $P D R_{i}$ for scenario 2 .

\footnotetext{
${ }^{8}$ In fact, LIKUID always outperforms RTE independently of the number of hops in a path when $R$ is equal or higher than 2.

${ }^{9}$ LIKUID $_{4}$ assigns the same total number of links for the path as $\mathrm{HbH}$ and RTE in scenario 2 . LIKUID $_{3}$ assigned the same total number of links for the path as $\mathrm{HbH}$ and RTE in scenario 1.
} 
The comparison of Fig. 9 and Fig. 6 shows that the energy consumption increases with the number of hops. Similar conclusions in terms of energy consumption and efficiency can be reached when comparing the different link scheduling schemes under scenario 2. LIKUID offers the possibility to flexibly utilize the shared links for retransmissions. This has a cost in terms of URA and total energy consumption under low link quality levels. Such cost increases with the number of hops. However, a close look to the energy efficiency $\left(E_{P M D}\right)$ shows that even if the number of hops increases, LIKUID is still more efficient when evaluating the energy per message correctly received at the destination node. In fact, $\mathrm{LIKUID}_{4}$ and $\mathrm{LIKUID}_{3}$ achieve the lowest $E_{P M D}$ levels.

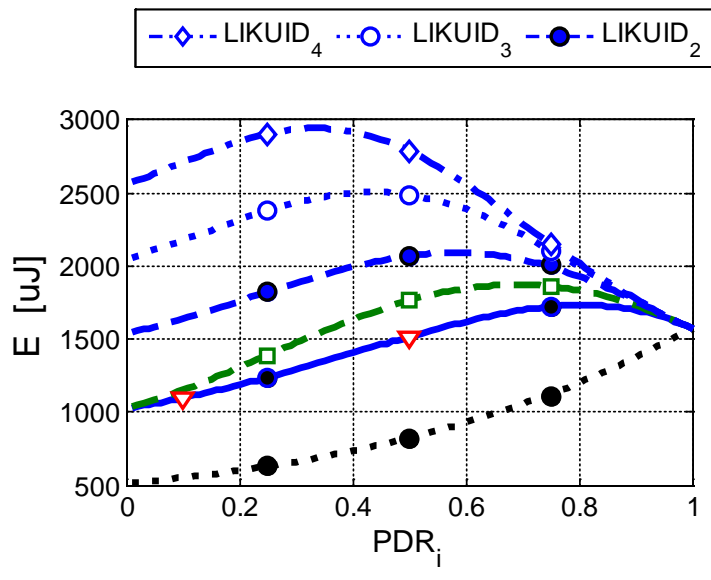

(a) Total Energy
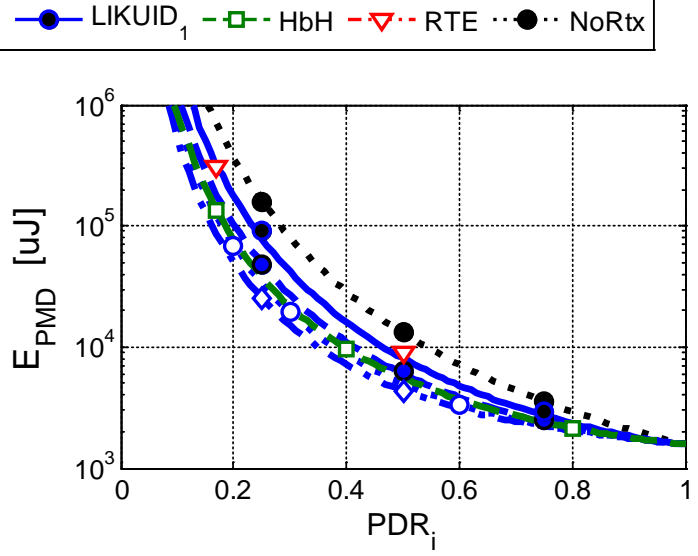

(b) Energy per message delivery

Fig. 9. Total energy consumption and average energy consumed per message correctly received at the destination node as a function of the $P D R_{i}$ for scenario 2.

Tables 2 and 3 show that increasing the number of hops does not have an impact on the number of blocked links per node. The only notable effects are: 1) more intermediate nodes benefit with LIKUID from the reduction in the number of blocked links compared to $\mathrm{HbH}$ and RTE when we decrease $R$, and 2) increasing the number of possible retransmissions using shared links (i.e. increasing $R$ from 3 to 4 ) increases the number of blocked links for all nodes in the path. Such increase helps though augment the $P D R_{E 2 E}$ and reduce the $D_{E 2 E}$ with respect to $\mathrm{HbH}$ and RTE.

Table 3 - Blocked links $(H=4)$

\begin{tabular}{c|ccccccc}
\hline \hline Node & LIKUID $_{4}$ & LIKUID $_{3}$ & LIKUID $_{2}$ & LIKUID $_{1}$ & HbH & RTE & NoRtx \\
\hline $\mathrm{A}$ & 5 & 4 & 3 & 2 & 2 & 2 & 1 \\
$\mathrm{~B}$ & 6 & 5 & 4 & 3 & 4 & 4 & 2 \\
$\mathrm{C}$ & 6 & 5 & 4 & 3 & 4 & 4 & 2 \\
$\mathrm{D}$ & 6 & 5 & 4 & 3 & 4 & 4 & 2 \\
$\mathrm{E}$ & 5 & 4 & 3 & 2 & 2 & 2 & 1 \\
\hline \hline
\end{tabular}

\subsection{Scenario 3}

Scenarios 1 and 2 assumed that all hops experience the same $P D R_{i}$. The third scenario simulates a more realistic scenario with variable $P D R_{i}$ per hop. The $P D R_{i}$ conditions have been simulated using the radio propagation model described in [19] and considering that nodes can be separated by 50 or $150 \mathrm{~m}$. With the simulation conditions reported in Section 6 , a distance between nodes of $50 \mathrm{~m}$ results in an average PDR equal to 0.953 . The average PDR decreases to 0.711 when the distance between nodes increases to $150 \mathrm{~m}$. All possible configurations of distance between hops (eight) have been simulated and evaluated for a path with $H=3$. The configurations are referenced with the possible combinations of values for $\left[d_{A B}, d_{B C}, d_{C D}\right] . d_{A B}, d_{B C}$ and $d_{C D}$ represent the distance between nodes A and B, B and C, and C and D respectively.

Table 4 shows the $P D R_{E 2 E}$ obtained for the different schemes and all possible configurations of distance between hops. First of all, it is important highlighting that Table 4 confirms the trends observed under scenarios 1 and 2. Under realistic simulation conditions, LIKUID improves again the reliability compared to existing schemes ( $\mathrm{HbH}$ and RTE). The results also show that when the path includes at least one long distance hop, $\mathrm{LIKUID}_{3}$ achieves a $P D R_{E 2 E}$ notably higher than the other schemes. LIKUID 2 experiences a slight degradation with respect to $\mathrm{LIKUID}_{3}$, but still significantly outperforms $\mathrm{HbH}$ and RTE. LIKUID is more robust because it can utilize more links for the long distance hops that experience lower PDR levels. On the other hand, $\mathrm{HbH}$ and RTE can only utilize a maximum of two links per hop independently of their link quality. It is also relevant noting that the achieved $P D R_{E 2 E}$ depends on the number of long distance hops in the path, but not on their position in the path. 
Table $4-P D R_{E 2 E}$ in scenario 3

\begin{tabular}{c|ccccc}
\hline \hline$\left[\mathrm{d}_{\mathrm{AB}}, \mathrm{d}_{\mathrm{BC}} \cdot \mathrm{d}_{\mathrm{CD}}\right]$ & LIKUID $_{3}$ & LIKUID $_{2}$ & HbH & RTE & NoRtx \\
\hline$[50,50,50]$ & 0.999 & 0.999 & 0.997 & 0.993 & 0.902 \\
\hline$[50,50,150]$ & 0.992 & 0.972 & 0.915 & 0.902 & 0.665 \\
{$[50,150,50]$} & 0.992 & 0.972 & 0.916 & 0.901 & 0.665 \\
{$[150,50,50]$} & 0.992 & 0.971 & 0.915 & 0.901 & 0.665 \\
\hline$[150,150,50]$ & 0.972 & 0.921 & 0.841 & 0.789 & 0.491 \\
{$[150,50,150]$} & 0.972 & 0.921 & 0.840 & 0.789 & 0.491 \\
{$[50,150,150]$} & 0.972 & 0.921 & 0.840 & 0.789 & 0.491 \\
\hline$[150,150,150]$ & 0.939 & 0.853 & 0.772 & 0.674 & 0.362 \\
\hline \hline
\end{tabular}

The results depicted in Table 5 also confirm the superior $D_{E 2 E}$ performance of LIKUID under realistic simulation conditions. It is interesting to note that the $D_{E 2 E}$ achieved with LIKUID $_{3}$, LIKUID $_{2}$ and RTE depends on the number of long distance hops in the path (the fewer the number of long distance hops, the smaller the delay). This is the case because each retransmission augments $D_{E 2 E}$ for these three schemes independently of the hop for which the retransmission was necessary. On the other hand, the delay performance of $\mathrm{HbH}$ does not depend on the number of long distance hops in the path, but mainly on the link quality of the last hop in the path. In fact, only the retransmissions on the last hop of the path increase the $D_{E 2 E}$ metric for $\mathrm{HbH}$.

Table 5 - $D_{E 2 E}[$ links] in scenario 3

\begin{tabular}{c|ccccc}
\hline \hline$\left[\mathrm{d}_{\mathrm{AB}}, \mathrm{d}_{\left.\mathrm{BC} \cdot \mathrm{d}_{\mathrm{CD}}\right]}\right.$ & LIKUID $_{3}$ & LIKUID $_{2}$ & HbH & RTE & NoRtx \\
\hline$[50,50,50]$ & 3.10 & 3.10 & 5.03 & 3.28 & 3.00 \\
\hline$[50,50,150]$ & 3.44 & 3.39 & 5.22 & 3.79 & 3.00 \\
{$[50,150,50]$} & 3.44 & 3.39 & 5.03 & 3.79 & 3.00 \\
{$[150,50,50]$} & 3.44 & 3.39 & 5.03 & 3.79 & 3.00 \\
\hline$[150,150,50]$ & 3.74 & 3.61 & 5.03 & 4.14 & 3.00 \\
{$[150,50,150]$} & 3.74 & 3.61 & 5.22 & 4.13 & 3.00 \\
{$[50,150,150]$} & 3.74 & 3.61 & 5.22 & 4.14 & 3.00 \\
\hline$[150,150,150]$ & 3.99 & 3.79 & 5.22 & 4.39 & 3.00 \\
\hline \hline
\end{tabular}

Table 6 reports the average percentage of links that have been utilized (out of all the assigned links for the path) under scenario 3. We can see that $\mathrm{LIKUID}_{3}$ utilizes the links similarly to $\mathrm{HbH}$ and RTE when there are no long distance hops in the path. When the number of such hops increases, $\mathrm{LIKUID}_{3}$ augments the utilization of assigned links with respect to HbH and RTE. This means that when the link quality degrades, $\mathrm{LIKUID}_{3}$ utilizes more the available links to conduct retransmissions, which ultimately benefits its $P D R_{E 2 E}$ performance as previously highlighted. The results in Table 6 show that it is actually LIKUID $_{2}$ the scheme that results in a higher utilization of the assigned links $\left(10 \%\right.$ more than LIKUID $\left._{3}\right)$. This is due to the fact that LIKUID 2 has one link less assigned to the complete path compared to LIKUID $_{3}$. In this case, LIKUID $_{2}$ needs to do a more intense utilization of the available links to conduct retransmissions when the link quality conditions degrade. However, its capacity to efficiently combat poor link quality conditions decreases with respect to $\mathrm{LIKUID}_{3}$ as the number of long distance hops in the path increases.

Table 6 - Average percentage of links that have been utilized in scenario 3

\begin{tabular}{c|ccccc}
\hline \hline$\left[\mathrm{d}_{\mathrm{AB}}, \mathrm{d}_{\mathrm{BC}} \cdot \mathrm{d}_{\mathrm{CD}}\right]$ & LIKUID $_{3}$ & LIKUID $_{2}$ & HbH & RTE & NoRtx \\
\hline$[50,50,50]$ & $51.7 \%$ & $62.1 \%$ & $51.6 \%$ & $51.6 \%$ & $96.7 \%$ \\
\hline$[50,50,150]$ & $57.7 \%$ & $68.7 \%$ & $55.9 \%$ & $55.5 \%$ & $96.7 \%$ \\
{$[50,150,50]$} & $57.6 \%$ & $68.1 \%$ & $54.5 \%$ & $54.1 \%$ & $88.5 \%$ \\
{$[150,50,50]$} & $57.5 \%$ & $67.7 \%$ & $53.1 \%$ & $52.7 \%$ & $80.0 \%$ \\
\hline$[150,150,50]$ & $62.8 \%$ & $72.4 \%$ & $55.7 \%$ & $53.8 \%$ & $74.0 \%$ \\
{$[150,50,150]$} & $63.1 \%$ & $73.4 \%$ & $56.9 \%$ & $55.6 \%$ & $80.1 \%$ \\
{$[50,150,150]$} & $63.2 \%$ & $73.9 \%$ & $58.3 \%$ & $57.0 \%$ & $88.5 \%$ \\
\hline$[150,150,150]$ & $68.0 \%$ & $77.3 \%$ & $59.2 \%$ & $55.9 \%$ & $74.0 \%$ \\
\hline \hline
\end{tabular}

The results depicted in Table 7 show that $\mathrm{LIKUID}_{3}$ results again in the larger URA. However, the impact of LIKUID on the URA reduces when $R$ decreases (i.e. $\mathrm{LIKUID}_{2}$ ). Table 7 also shows that URA increases when the long distance hops are located in the first hops of the path. In this case, retransmissions are usually necessary to progress with the transmission of the message towards the destination node. The nodes that are closer to the destination will be unnecessarily in reception mode until such 
retransmissions are successful. LIKUID increases the utilization of links under scenario 3 in order to accommodate the necessary retransmissions that benefit the final $P D R_{E 2 E}$. Despite these additional retransmissions, Table 8 shows that LIKUID does not increase the average energy per message correctly received at the destination node $\left(E_{P M D}\right)$. In fact, LIKUID can even slightly decrease $E_{P M D}$ compared to existing schemes. Table 8 also shows that the reduction in $E_{P M D}$ achieved with LIKUID compared with the other schemes is higher as the number of long distance hops in the path increases. This is the case because LIKUID increases the number of messages delivered to the destination node, and therefore decreases the energy consumed per delivered message.

Table 7 - URA metric in scenario 3

\begin{tabular}{c|ccccc}
\hline \hline$\left[\mathrm{d}_{\mathrm{AB}}, \mathrm{d}_{\mathrm{BC}} \cdot \mathrm{d}_{\mathrm{CD}}\right]$ & LIKUID $_{3}$ & LIKUID $_{2}$ & $\mathrm{HbH}$ & $\mathrm{RTE}$ & NoRtx \\
\hline$[50,50,50]$ & 0.10 & 0.10 & 0.10 & 0.09 & 0 \\
\hline$[50,50,150]$ & 0.10 & 0.10 & 0.10 & 0.07 & 0 \\
{$[50,150,50]$} & 0.44 & 0.39 & 0.29 & 0.26 & 0 \\
{$[150,50,50]$} & 0.78 & 0.68 & 0.48 & 0.45 & 0 \\
\hline$[150,150,50]$ & 1.05 & 0.87 & 0.67 & 0.54 & 0 \\
{$[150,50,150]$} & 0.73 & 0.61 & 0.48 & 0.38 & 0 \\
{$[50,150,150]$} & 0.42 & 0.35 & 0.29 & 0.22 & 0 \\
\hline$[150,150,150]$ & 0.99 & 0.79 & 0.67 & 0.46 & 0 \\
\hline \hline
\end{tabular}

Table 8 - $E_{P M D}[\mu \mathrm{J}]$ in scenario 3

\begin{tabular}{c|ccccc}
\hline \hline$\left[\mathrm{d}_{\mathrm{AB}}, \mathrm{d}_{\mathrm{BC}} \cdot \mathrm{d}_{\mathrm{CD}}\right]$ & LIKUID $_{3}$ & LIKUID $_{2}$ & HbH & RTE & NoRtx \\
\hline$[50,50,50]$ & 1225 & 1225 & 1226 & 1229 & 1268 \\
\hline$[50,50,150]$ & 1377 & 1394 & 1444 & 1457 & 1721 \\
{$[50,150,50]$} & 1388 & 1398 & 1422 & 1434 & 1590 \\
{$[150,50,50]$} & 1400 & 1403 & 1401 & 1413 & 1455 \\
\hline$[150,150,50]$ & 1572 & 1597 & 1613 & 1661 & 1843 \\
{$[150,50,150]$} & 1566 & 1603 & 1634 & 1700 & 1972 \\
{$[50,150,150]$} & 1554 & 1598 & 1658 & 1725 & 2154 \\
\hline$[150,150,150]$ & 1758 & 1835 & 1865 & 2021 & 2497 \\
\hline \hline
\end{tabular}

\subsection{Scenario 4}

The fourth scenario extends the third scenario and considers that the first node in the path (node A) is a mobile node. In the simulated scenario, node A moves around the coverage area of node B at a speed of $1 \mathrm{~m} / \mathrm{s}$ and following the random waypoint mobility model [17]. The maximum distance between nodes A and B has been set to $150 \mathrm{~m}$. The fourth scenario also considers variable $P D R_{i}$ levels at each hop, and distances of 50 or $150 \mathrm{~m}$ between nodes $\mathrm{B}$ and $\mathrm{C}$, and between nodes $\mathrm{C}$ and $\mathrm{D}$.

Fig. 10 represents the results obtained for all the metrics under scenario 4 . The figure depicts the average values and the $5^{\text {th }}$ and $95^{\text {th }}$ percentiles. Fig. 12 demonstrates that the proposed link scheduling scheme outperforms existing solutions even under the presence of mobile nodes. The configuration of LIKUID with $R$ equal to $3\left(\operatorname{LIKUID}_{3}\right)$ achieves the highest $P D R_{E 2 E}$ (Fig. 10a) and the smallest $D_{E 2 E}$ (Fig. 10b). LIKUID 3 also augments the average utilization of the available links compared to HbH and RTE (Fig. 10c), but increases the URA (Fig. 10d). As it was previously the case, such higher URA is the cost incurred by the scheduling flexibility embedded in LIKUID and that is at the origin of its higher reliability and latency performance. However, LIKUID $_{3}$ is again the most efficient scheme for delivering messages to the destination node and results in the lower $E_{P M D}$ value (Fig. 10e). Similar trends to that reported for scenario 3 are also observed under scenario 4 with a mobile node. In particular, the gains achieved by LIKUID with respect to existing schemes augment when the link quality conditions $\left(P D R_{i}\right)$ decrease. For example, $\mathrm{LIKUID}_{3}$ improves the $P D R_{E 2 E}$ with respect to $\mathrm{HbH}$ by $2 \%$ under the configuration [50,50]. The improvement increases to $14 \%$ under the configuration [150,150]. LIKUID also experiences a smaller $P D R_{E 2 E}$ reduction when the link quality conditions degrade compared to existing schemes. For example, the $P D R_{E 2 E}$ performance of $\mathrm{LIKUID}_{3}$ only decreases by $3.4 \%$ when comparing the best $([50,50]$ configuration) and worst $([150,150]$ configuration) link quality conditions. On the other hand, the $P D R_{E 2 E}$ performance of $\mathrm{HbH}$ and RTE decreases by $15.4 \%$ and $21.4 \%$ respectively under the same conditions. The capacity exhibited by LIKUID to combat poor link quality conditions is highly relevant for critical applications, such as the industrial ones, that demand very high reliability levels. The results also show that LIKUID is capable to guarantee high $P D R_{E 2 E}$ levels to mobile nodes. So as long as the mobile nodes do not leave the communications range of their connecting nodes, LIKUID does not need to reschedule the links since it can maintain high $P D R_{E 2 E}$ levels. 


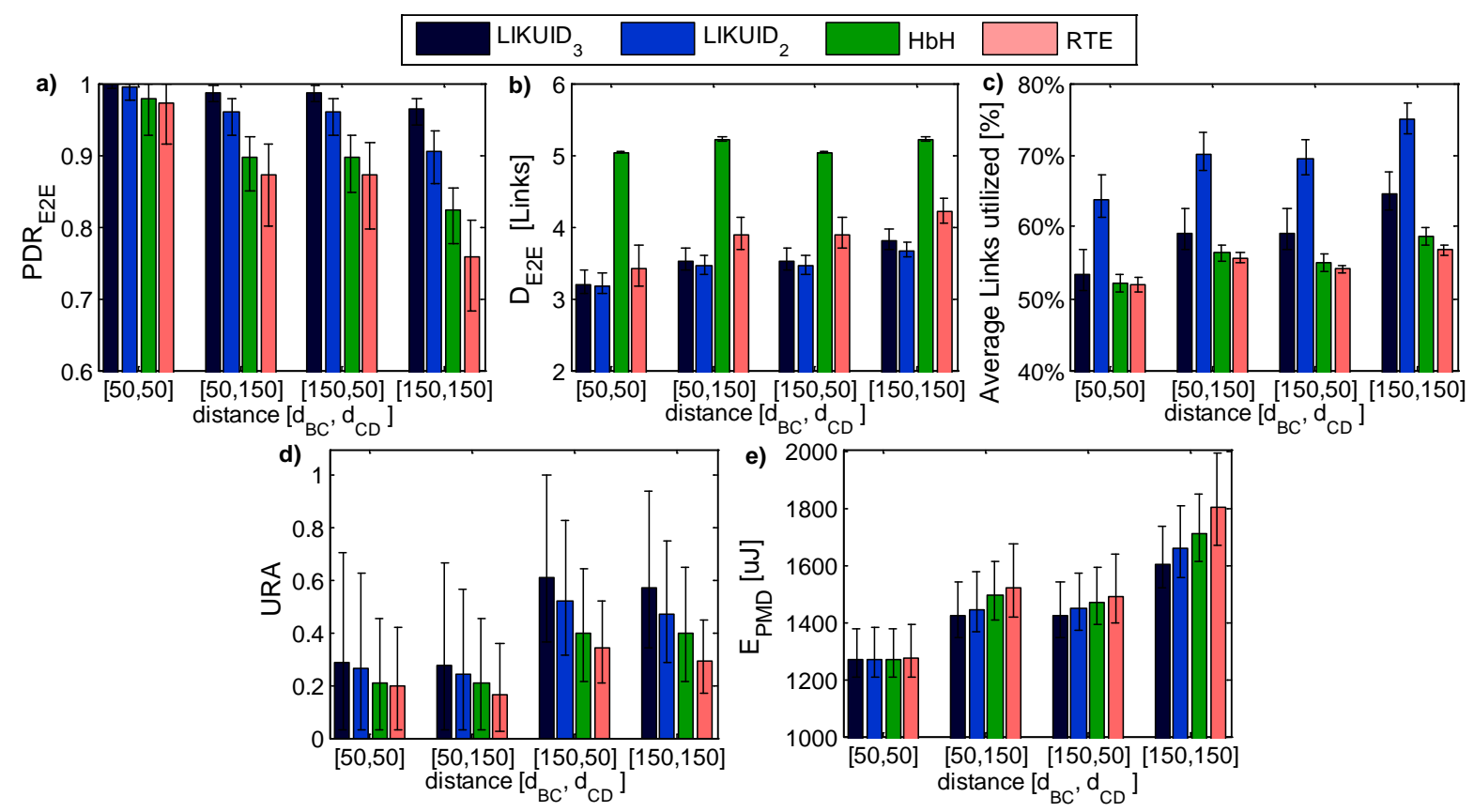

Fig. 10. Results (average, 5th and 95th percentiles) obtained under scenario 4.

\section{Conclusions}

This paper has presented and evaluated a novel link scheduling scheme for industrial wireless sensor networks with strict reliability and latency requirements. The proposed scheme proposes the use of shared links between nodes that are part of a given path or multi-hop route. The messages transmitted over the route act as a virtual token to identify which node should utilize the shared links at each point in time. The use of shared links and virtual tokens allows for a more flexible use of the available links that can take into account the link quality conditions and the need for retransmissions. The proposed link scheduling scheme is here applied to IWSNs, but it is important emphasizing that it can be used in other centralized TDMA-based multi-hop wireless networks.

The proposed link scheduling scheme has been thoroughly evaluated and its performance has been compared against existing schemes. To this aim, this paper has presented analytical expressions of the reliability $\left(P D R_{E 2 E}\right)$ and latency $\left(D_{E 2 E}\right)$ performance that can be used for a first performance estimate. The evaluation has been extended using numerical evaluations and simulations under different scenarios and operating conditions. The obtained results clearly demonstrate that the proposed link scheduling scheme significantly improves the reliability and end-to-end delay of the existing schemes when utilizing the same total number of links assigned to a path or multi-hop route. This trend has been shown to be independent of the number of hops in the route, the link quality conditions per hop, and the presence of mobile nodes in the route. The proposed scheme also efficiently utilizes the available resources. In particular, it increases the utilization of the assigned links and reduces the energy consumed per message correctly delivered to the destination node. All these benefits are obtained at the cost of increasing the number of blocked links and the time a node is unnecessarily in reception mode in case it should receive a message. These drawbacks can be deemed relatively minor considering the reliability and latency gains achieved with the proposed link scheduling scheme. In any case, they can be reduced by decreasing the number of shared links used for retransmissions $(R)$. Decreasing $R$ also reduces the total number of links assigned for a given path or multi-hop route, but it has also an impact on the reliability. The study has also shown that it is possible to maintain the reliability levels offered by existing schemes such as $\mathrm{HbH}$ or RTE while reducing the total number of links assigned to a route (e.g. with LIKUID $_{1}$ ). In this case, the proposed scheme can free valuable resources that can be used for other functions in the network. The proposed link scheduling scheme is then a valuable solution to improve the reliability and latency in industrial wireless sensor networks with strict application requirements, and to efficiently utilize the available resources (i.e. links or bandwidth and energy).

\section{Acknowledgment}

This work was supported in part by the Spanish Ministry of Economy and Competitiveness and FEDER funds under the project TEC2014-57146-R, and by the Local Government of Valencia with reference ACIF/2013/060. 


\section{References}

[1] Hancke G, Gungor V, Hancke G (2014) Guest editorial special section on industrial wireless sensor networks. IEEE Transactions on Industrial Informatics, vol. 10, no. 1, pp. 762-765. doi: 10.1109/TII.2013.2280433

[2] IEC 62591 Ed. 1.0: Industrial communication networks -Wireless communication network and communication profilesWirelessHART TM, IEC, 2010.

[3] Wireless Systems for Industrial Automation: Process Control and Related Applications, ISA-100.11a-2009 Standard, 2009.

[4] Dobslaw F, Zhang T, Gidlund M (2014) End-to-end reliability-aware scheduling for wireless sensor networks. IEEE Transactions on Industrial Informatics, (available as early access article). doi: 10.1109/TII.2014.2382335.

[5] Petersen S, Carlse (2011) WirelessHART Versus ISA100.11a: The Format War Hits the Factory Floor. IEEE Industrial Electronics Magazine, vol. 5, no. 4, pp. 23-34. doi:10.1109/MIE.2011.943023.

[6] Suriyachai P, Roedig U, Scott A (2012) A Survey of MAC Protocols for Mission-Critical Applications in Wireless Sensor Networks. IEEE Communications Surveys \& Tutorials, vol. 14, no. 2, pp. 240-264. doi:10.1109/SURV.2011.020211.00036.

[7] Sgora A, Vergados DJ, Vergados DD (2015) A Survey of TDMA Scheduling Schemes in Wireless Multihop Networks. ACM Computing Surveys, vol. 47, no. 3, art. 53, pp. 1-39. doi:10.1145/2677955.

[8] Mathad KS, Mangalwede SR (2015) Scheduling Approaches and Routing Protocols in Wireless Mesh Networks-A Survey. International Journal of Advanced Research in Computer and Communication Engineering, vol. 4, no. 8, pp. 307-310. doi:10.17148/IJARCCE.2015.4865.

[9] Saifullah A, Xu Y, Lu C, Chen Y (2015) Distributed Channel Allocation Protocols for Wireless Sensor Networks. IEEE Transactions on Parallel and Distributed Systems, vo. 25, no. 9, pp. 2264-2274. doi:10.1109/TPDS.2013.185.

[10] Rhee I, Warrier A, Min J, Xu L (2009) DRAND: Distributed Randomized TDMA Scheduling for Wireless Ad Hoc Networks. IEEE Transactions on Mobile Computing, vol. 8, no. 10, pp. 1384-1396. doi:10.1109/TMC.2009.59.

[11]Zand P, Chatterjea S, Ketema J, Havinga P (2012) Distributed Scheduling Algorithm for Real-time (D-SAR) Industrial Wireless Sensor and Actuator Networks. Proc. of the 17th Conference on Emerging Technologies \& Factory Automation (ETFA'12). doi:10.1109/ETFA.2012.6489719.

[12] Li Y, Zhang H, Huang Z, Albert M (2014) Optimal link scheduling for delay-constrained periodic traffic over unreliable wireless links. Proc. of the 33th IEEE Conference on Computer Communication (INFOCOM'14). doi:10.1109/INFOCOM.2014.6848081.

[13]Zhang S, Zhang G, Yan A, Xiang Z, Ma T (2013) A highly reliable link scheduling strategy for WirelessHART networks. Proc. of the 2013 IEEE International Conference on Advanced Technologies for Communication (ATC). doi:10.1109/ATC.2013.6698073.

[14] Saifullah A, Xu Y, Lu C, Chen Y (2010) Real-Time Scheduling for WirelessHART Networks. Proc. of the 31st IEEE RealTime Systems Symposium (RTSS). doi:10.1109/RTSS.2010.41.

[15] Dang K, Shen JC, Dong LD, Xia YX (2013) A Graph Route-Based Superframe Scheduling Scheme in WirelessHART Mesh Networks for High Robustness. Wireless Personal Communications, vol. 71, no. 4, pp. 2431-2444. doi:10.1007/s11277-0120946-2.

[16] Suriyachai P, Roedig U, Scott A (2009) Implementation of a MAC protocol for QoS support in wireless sensor networks. Proc. of the IEEE International Conference on Pervasive Computing and Communications (PerCom 2009). doi:10.1109/PERCOM.2009.4912839.

[17] Broch D, Maltz A, Johnson DB, Hu YC, Jetcheva J (1998) A performance comparison of multi-hop wireless ad hoc network routing protocols. Proc. of the 4th ACM/IEEE International Conference on Mobile Computing and Networking (Mobicom98). doi:10.1145/288235.288256.

[18] XDM2510H, 2.4 GHz IEEE 802.15.4 WirelessHART compliant radio module, RF Monolithics, Mar. 2011.

[19] Tanghe E, Joseph W, et al. (2008) The industrial indoor channel: large-scale and temporal fading at 900, 2400, and 5200 MHz. IEEE Transactions on Wireless Communications, vol. 7, no. 7, pp. 2740-2751. doi:10.1109/TWC.2008.070143. 